\title{
Light distortion and spherical aberration for the accommodating and nonaccommodating eye
}

Rute Macedo-de-Araújo

Helena Ferreira-Neves

Laura Rico-del-Viejo

Sofia C. Peixoto-de-Matos

José Manuel González-Méijome 


\title{
Light distortion and spherical aberration for the accommodating and nonaccommodating eye
}

\author{
Rute Macedo-de-Araújo, Helena Ferreira-Neves, Laura Rico-del-Viejo, Sofia C. Peixoto-de-Matos, and José \\ Manuel González-Méijome* \\ University of Minho, School of Science, Department and Center of Physics, Clinical and Experimental Optometry Research Lab, Gualtar 4710-057, \\ Braga, Portugal
}

\begin{abstract}
To evaluate how different amounts of induced spherical aberration (SA) affect the light distortion (LD) phenomena, tests were performed using an experimental device to measure the distortion (haloes, glare, and so on) of a point source. To simulate the effect of SA, eight different phase plates between +0.300 and $-0.300 \mu \mathrm{m}$ of SA for a 5-mm aperture were used in a random and double-masked experimental design. Measurements were performed at a distance of $2 \mathrm{~m}$ in a darkened room in 10 eyes of five subjects with a mean age of $26.4 \pm 6.1$ years and a mean refractive error of $-0.50 \pm 0.70 \mathrm{D}$. Data were obtained with natural pupil and after pupil dilatation. The measurements with this experimental system showed a significant increase in all distortion parameters with cycloplegia for the phase plates with the higher positive SA $(+0.300$ and $+0.150 \mu \mathrm{m})$. The disturbance index increased from $14.86 \pm 6.12 \%$ to $57.98 \pm 36.20 \%(p<0.05)$ with the $+0.300 \mu \mathrm{m}$ plate. The same effect was observed through at a much lower rate when the eye could accommodate. Plates inducing negative SA did not change the LD compared to the control condition without induction of SA or even decreased the effect of distortion. Pupillary dilation and cyclopegia led to a significant increase in the size of the LD when increasing values of SA were induced. Accommodation and pupillary constriction are capable of compensating the degradation of the optical quality induced. () 2016 Society of Photo-Optical Instrumentation Engineers (SPIE) [DOI: 10.1117/1.JBO.21.7 .075003]
\end{abstract}

Keywords: light distortion; glare; haloes; pupil size; spherical aberration.

Paper 150501RR received Jul. 25, 2015; accepted for publication Jun. 13, 2016; published online Jul. 7, 2016.

\section{Introduction}

There is a growing interest in evaluating visual function under low lighting conditions and in the presence of light sources that originate glare, haloes, starbursts, and other light distortion (LD) effects previously described in the literature. ${ }^{1}$ Several clinical procedures, such as refractive surgery and intraocular lens implantation, might significantly increase the complaints of patients referring haloes and other disturbance phenomena, leading to visual dissatisfaction. ${ }^{2}$ Brito et al. $^{3}$ recently studied LD in patients implanted with multifocal intraocular lenses (IOL) and showed that the night vision disturbances (NVD) are significantly higher when compared with the monofocal control group. Ocular aberrations and other factors that degrade the optical quality of the eye produce photic phenomena such as haloes, starbursts, scattering, and others, when observing a light source. However, such terms need to be clarified, as they are often confounded by patients and clinicians. As Fan-Paul et al. ${ }^{1}$ stated, patients are not able to differentiate the terms relating to NVD and self-report "glare" complaints for describing their night vision difficulties. Technically, the term "glare" only refers to a light source, and the physical phenomena that causes decrease in quality of vision is, in fact, the combination of at least three major complaints: glare disability, which is the term used to define any subjective reduction of visual performance due to a glare source, image degradations, i.e., defined as the alteration of objects' shape or size (also known as haloes and starbursts), and contrast sensitivity that is reduced under these circumstances. As it is difficult to differentiate the type of disturbance observed, some authors suggest the concept of "LD" as a general concept that contemplates all the previous phenomena. ${ }^{4}$ Although all these disabilities are seen under scotopic or mesopic conditions, they can occur in subjects with good vision under photopic conditions. ${ }^{1}$ The source may be very different: some disturbances can derivate from light dispersion phenomena, which lead to the glare incapacity (retinal straylight) and scattering; others can be due to LD characterized by image distortion under low lighting conditions such as starbursts (star shaped) and haloes; and others can be due to the decrease of contrast sensitivity under scotopic and mesopic conditions.

Traditionally, the description of such phenomena has been done by means of pictorial representations, ${ }^{1}$ and more recently by visual simulators available over the internet. ${ }^{5}$ Other approaches have aimed to use software facilities to work on a computer screen, with several limitations to mimic real-world situations. For example, systems working only on computer screens ${ }^{6,7}$ are limited by the luminous intensity output of cathode ray tubes (CRT) screens, liquid crystal displays (LCD), and light-emitting diodes (LEDs) screens. It is difficult to use white spots on a CRT, LCD, or LED screen to simulate certain effects that are visible with real sources of light such as lines arising out from the source such as the headlights of cars, street lighting, or stars in the dark sky (starburst effect). We have recently described a new system to measure the LD based on a hardware presenting bright stimuli against a dark background combined 
with software for stimuli presentation and data analysis and storage. ${ }^{8}$

This study aims to quantify and understand how different amounts of positive and negative spherical aberrations (SAs) affect the phenomenon of LD as well as its morphology, under normal accommodation and after cycloplegia, and determine if this device is sensitive enough to measure those effects.

\section{Material and Methods}

\subsection{Sample}

Five healthy volunteers ( 4 female, 1 male, 10 eyes) participated in the study. All had normal ocular and general health, with ages ranging from 23 to 37 years ( $26.4 \pm 6.1$ years). Inclusion criteria required that the subjects had no complaints of dry eye, did not wear contact lenses, and had no ocular pathologies or had a previous ocular surgery.

All subjects underwent a full optometric examination including: objective and subjective refraction using an end-point criterion of maximum plus for the best visual acuity, pupil diameter measurement (NeurOptics ${ }^{\circledR} \mathrm{VIP}^{\mathrm{TM}}-200$, California), and whole eye wavefront aberrometry using a Harmann-Shack aberrometer (IRX3, Imagine Eyes, Orsay, France). Measurements were done before and after the instillation of cycloplegic with tropicamide $1 \%$ (Tropicil, Edol, Portugal). For all the subjects, two drops of cycloplegic were instilled (the second one 5 min after the first). Then the measurements were done $30 \mathrm{~min}$ after the instillation of the first drop.

The protocol of the study was reviewed and approved by the Ethics Subcommittee for Health and Life Sciences of University of Minho. Following the tenets of the Declaration of Helsinki, all subjects signed an informed consent after the nature and possible consequences of the study had been explained.

\subsection{Procedure}

Measurements of LD were performed using an experimental device-LD Analyzer (LDA, CEORLab, Portugal) that allows to analyze the light distortion index (LDI, \%), the radius ( $\mathrm{mm}$ ) of the circle that best fits the distortion [best-fit circle radius $\left.\left(\mathrm{BFC}_{\mathrm{rad}}\right)\right]$, and the irregularity of the distortion that is given by $\mathrm{BFC}$ irregularity $\left(\mathrm{BFC}_{\text {Irreg }}\right)(\mathrm{mm})$ and $\mathrm{SD}$ of $\mathrm{BFC}_{\text {Irreg }}$ $\left(\mathrm{BFCI}_{\text {rregSD }}\right)$, which indicates, for each meridian, the difference between the distortion and the circle that best fits it, and its standard deviation (SD), respectively. These indices will be explained below in detail. Eight different phase plates were used to simulate the effect of spherical Seidel aberration. The SA induced ranged from +0.300 to $-0.300 \mu \mathrm{m}$ for a $5-\mathrm{mm}$ pupil size [Plate $+1(+0.039 \mu \mathrm{m})$; Plate $+2(+0.075 \mu \mathrm{m})$; Plate $+3(+0.150 \mu \mathrm{m})$; Plate $+4(+0.300 \mu \mathrm{m})$; Plate $-1(-0.039 \mu \mathrm{m})$; Plate $-2 \quad(-0.075 \mu \mathrm{m}) ; \quad$ Plate $-3 \quad(-0.150 \mu \mathrm{m}) ; \quad$ Plate-4 $(-0.300 \mu \mathrm{m})]$. Both the subjects and the investigator doing the LDA measures were masked for the plate in use in each trial and the eight plates were randomly presented in the following order for all the subjects: without plate (baseline measure); Plate-1, Plate +4 , Plate-2, Plate +3 , Plate-3, Plate +2 , Plate-4, and Plate +1 . As stated before, the data were obtained first with natural pupil and normal accommodation and then after instillation of cycloplegic (Tropicil, Edol, Portugal). All patients did a trial with the instrument to adapt to the methodology before the measuring session. All measures were done in both eyes in random order, under monocular conditions. Examination was performed in a dark room with the subjects adapted to the dark before LD examination. The graphical and numerical information of the distortion caused by each plate was obtained by exporting images from the LDA software for each measure. ${ }^{8}$

LD was analyzed with an experimental prototype, the LDA (CEORLab, University of Minho, Portugal), which provides a comprehensive number of metrics to characterize the condition, and proved to be robust in measuring NVD in young healthy eyes and also in elderly patients. ${ }^{3,8,9}$ It consists of an electronic black board with a central light source (LED) surrounded by 240 small LED sources distributed in 24 semimeridians with a minimum angular separation of $15 \mathrm{deg}$. In this experiment, an angular separation of $30 \mathrm{deg}$ was considered to shorten the exam time without significant loss of information. ${ }^{9}$ Figure 1 (left) shows the layout arrangement of the central white LEDs and the surrounding smaller white LEDs. The technical specifications of the LEDs can be consulted in previously published work from Linhares et al. ${ }^{8}$

The subject was at a distance of $2.0 \mathrm{~m}$ in a darkened room and was instructed to always fixate on the central LED while doing the measurements. The total area under analysis covers $4.6 \mathrm{deg}$ of field. The physical (electronic board) display device is connected to a PC computer via USB connection. The subject being evaluated provides feedback to the system through a PC mouse. Peripheral stimuli are presented around the central source of light from the inner to the outer part of the field at random times from 250 to $750 \mathrm{~ms}$. Twelve semimeridians in 30-deg steps were explored in random order, with the "inout" routine, which refers to the strategy where the radial LEDs turn-on and turn-off sequentially from center to periphery. During the exam, when the subject sees the peripheral stimulus, he/she presses the mouse control and the system automatically evaluates the next semimeridian. All the semimeridians are examined three times at the same measurement. If the SD of the three measurements in each semimeridian is above $20 \%$ of the mean value, the device automatically repeats the measurements in those semimeridians until it reaches values of SD below $20 \%$ of the mean. After data collection and storage, a software tool calculates indices that determine the size, shape, and regularity of the distortion surrounding the central source of light. With this routine, the time of measurement is about $51.52 \pm 6.85 \mathrm{~s}$. When the distortion becomes larger the exam can be slower, but never exceeds $1 \mathrm{~min}$ and $20 \mathrm{~s}$ in this case.

The LDI is calculated as the ratio of the area of points missed by the subject and the total area explored and is expressed as a percentage $(\%)$. The higher values of distortion are interpreted as the lower ability to discriminate surrounding small stimuli that are hidden by the distortion induced from the central source of light.

The $\mathrm{BFC}_{\mathrm{rad}}$ is defined as the circle that best fits to the distortion area resulting from the linear binding of all external points not seen by the subject along each meridian. This parameter is expressed in millimeters and is linearly related to LDI parameter.

The irregularity of the distortion area is derived as the deviation of the actual polygonal shape obtained from the $\mathrm{BFC}$ fit and is called the BFC irregularity $\left(\mathrm{BFC}_{\text {Irreg }}\right)$. The $\mathrm{BFC}_{\text {IrregSD }}$ measures how asymmetric the departure of the actual limits of the distortion from the perfect circular shape of the $\mathrm{BFC}$ is. Together $\mathrm{BFC}_{\text {Irreg }}$ and $\mathrm{BFC}_{\text {IrregSD }}$ can be interpreted as the deviation of the actual distortion from a perfectly rotational symmetric shape. The higher the value of this parameter, 

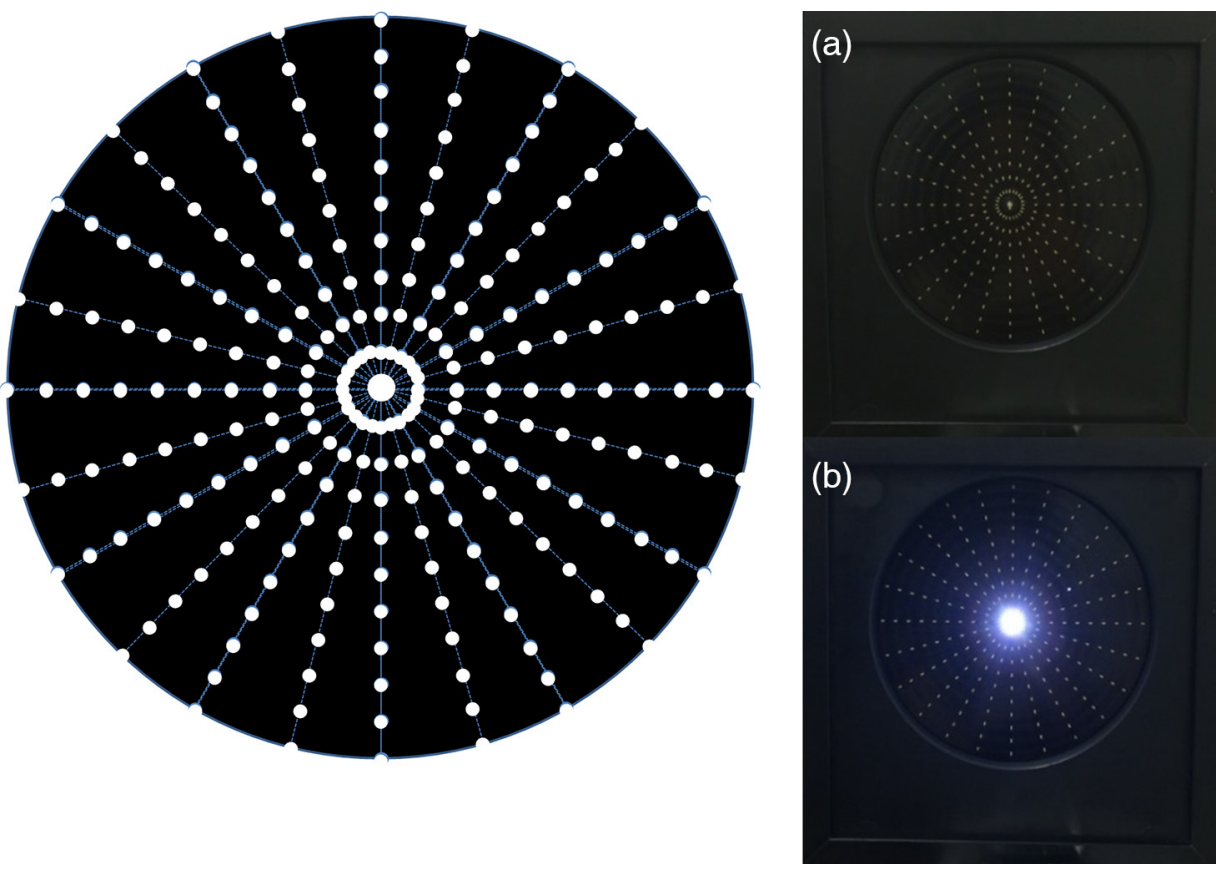

Fig. 1 Illustration of the distribution of main central source of light and peripheral stimuli in accordance with an exemplary embodiment of the present invention. On the right: the experimental device LDA with the central LED light (a) turned OFF and (b) turned ON at minimum intensity also with one peripheral LED turned ON.

the larger the deviation from a circular shape and it is expressed in millimeters, as shown in Fig. 1.

Once the subject was in front of the screen with the head on a chin-rest, the test began. Every time the subject saw a stimulus, they had to press the button that gives feedback to the central unit. A demonstration test was run before first measures for each subject.

\subsection{Statistical Analysis}

Both eyes from the same subject have been used considering that the interocular correlation between the LD at baseline was weak (Spearman rho $=0.456)$. Statistical analysis has been conducted using SPSS v21.0 (IBM Inc. Illinois). Considering the limited sample size, nonparametric statistics were applied. For the multiple comparison between the LD at baseline and with each of the plates (LDI, $\mathrm{BFC}_{\text {Irreg }}$, and $\left.\mathrm{BFC}_{\text {IrregSD }}\right)$, a Friedman test with posthoc correction has been applied. For pairwise comparison between pairs of plates or between baseline measures and each plate, Wilcoxon signed ranks test has been applied considering the related nature of the data. The level of statistical significance has been set at $p<$ 0.05 and for multiple comparisons for $p<0.0055(0.05 / 9)$.

\section{Results}

Average manifest spherical equivalent refraction was of $-0.50 \pm 0.70 \mathrm{D}$. The average value of the fourth-order SA in the tested eyes without and with the instillation of tropicamide were $+0.038 \pm 0.020$ and $+0.032 \pm 0.030 \mu \mathrm{m}$ for a $4-\mathrm{mm}$ pupil size, respectively, and $+0.160 \pm 0.100$ and $+0.297 \pm$ $0.350 \mu \mathrm{m}$ for the natural maximum round pupil, respectively. The maximum round pupil was $6.06 \pm 0.71 \mathrm{~mm}$ for the normal eye and $7.15 \pm 0.62 \mathrm{~mm}$ for the eye under cycloplegic effect.
As observed in Fig. 2, the measures with this experimental system showed a significant increase in all parameters when measured under tropicamide effect. These differences between the accommodative and nonaccommodative eyes are more noticeable in the three parameters studied with the phase plates corresponding to the highest positive SA: Plate $+4(+0.300 \mu \mathrm{m})$ and Plate $+3(+0.150 \mu \mathrm{m})$. On the other hand, the phase plates that induce negative SAs cause a smaller or no increase in the distortion size and its irregularity. There were statistically significant differences between the values with and without the instillation of tropicamide for most plates. In Figs. 2(a) and 2(b), which show the values obtained for $\mathrm{LDI}$ and $\mathrm{BFC}_{\mathrm{rad}}$, respectively, there were significant differences between the two situations in all plates except for Plate $+1(+0.0385 \mu \mathrm{m})$. The $\mathrm{BFC}_{\text {IrregSD }}$ [Fig. 2(c)] seems to be less affected by the instillation of tropicamide, with significant differences only in Plate-4 $(-0.300 \mu \mathrm{m} ; p<0.05)$ and Plate $+3(+0.150 \mu \mathrm{m}, p<0.01)$.

Figure 3 shows graphical examples of size and shape of the LD produced by the different plates with and without tropicamide. These images exemplify the actual size and shape of each experimental condition, and the values of $\mathrm{LDI}, \mathrm{BFC}_{\mathrm{rad}}$, and $\mathrm{BFC}_{\text {IrregSD }}$ are shown in each image. Comparing measures without and with cycloplegia, there is an apparent improvement with the Plate-3 $(-0.150 \mu \mathrm{m})$, Plate-2 $(-0.075 \mu \mathrm{m})$, and Plate-1 $(-0.039 \mu \mathrm{m})$ compared to the baseline image. The opposite happens with increasing positive SA, with higher distortion, especially when the eye is under cycloplegic effect.

Tables 1-3 show the differences ( $p$ values) between plates, with and without the instillation of cyclopegic drops. On these tables, the comparisons between plates for natural pupil and cycloplegic are shown separately and these two exam conditions are not compared here. The comparison between the measures without and with cycloplegia is shown in Figs. 2(a)-2(c). As illustrated in Fig. 2, there are larger differences between the 


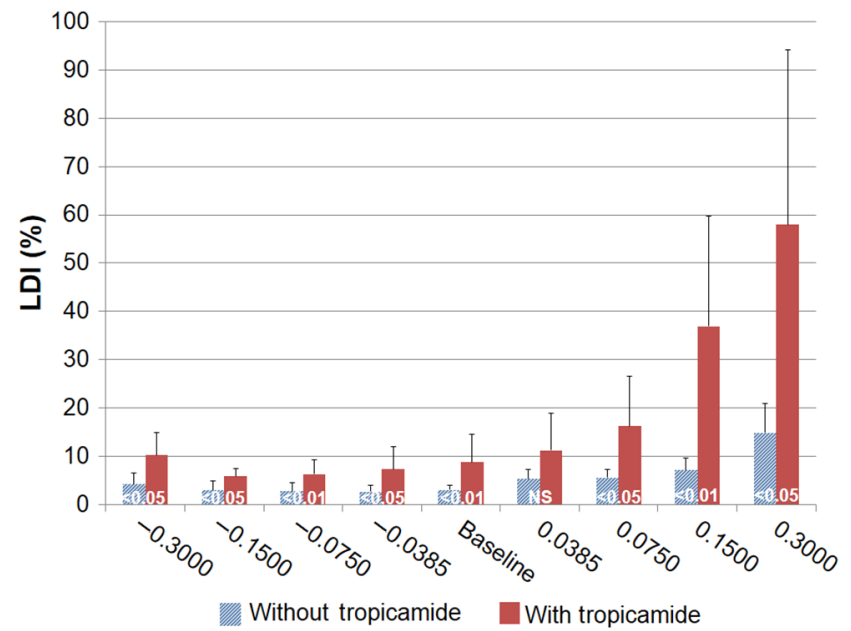

(a)

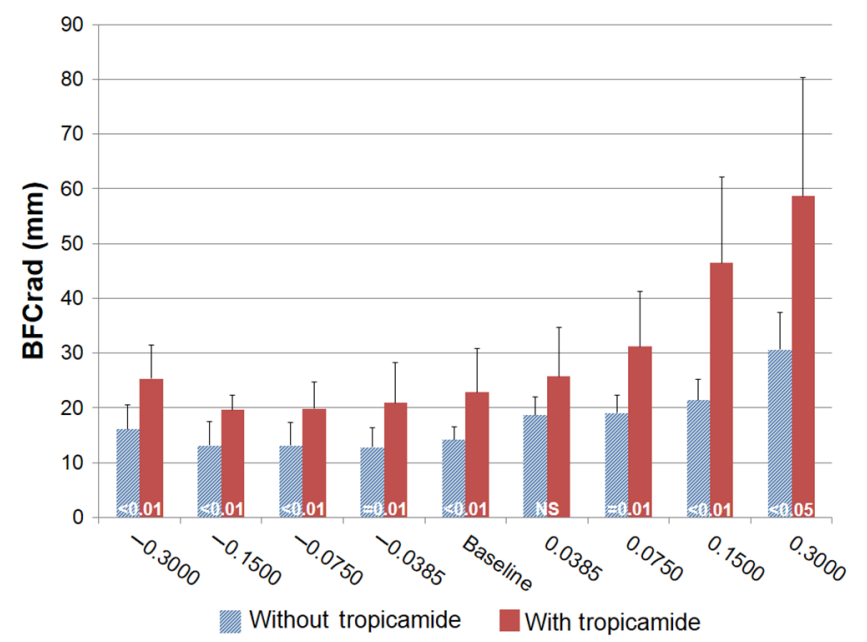

(b)

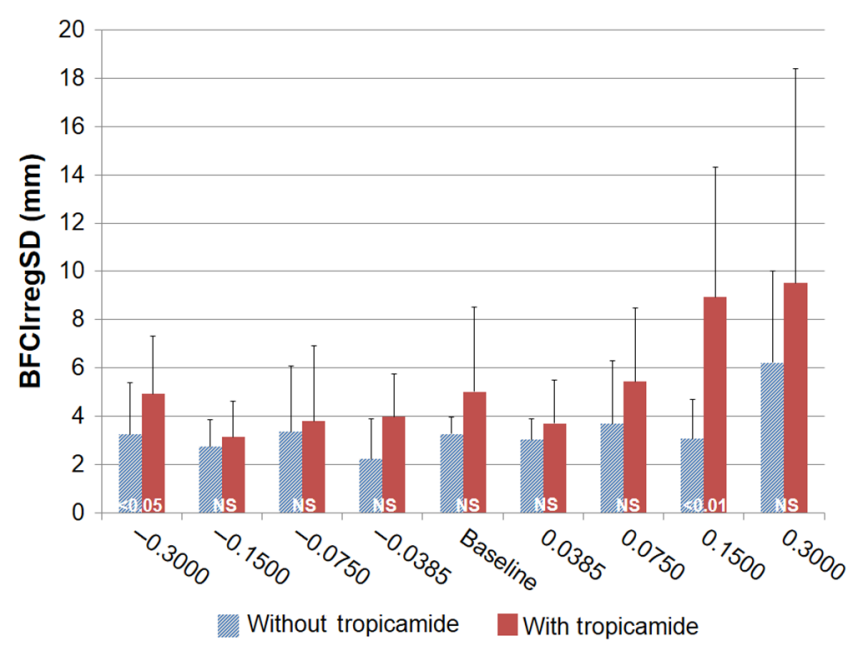

(c)

Fig. 2 Measurements of (a) LDI, (b) BFC $\mathrm{Bad}_{\text {, }}$, and (c) BFC $\mathrm{IrregSD}_{\text {, with }}$ eight phase plates that induce different levels of SA. The measures of the 10 eyes with and without tropicamide are presented. NS, no significant differences were found. baseline measure and the phase plates that induce positive SA, both with and without tropicamide, than with the phase plates that induce negative SA. This is shown in Tables 1 and 2 (LDI and $\mathrm{BFC}_{\mathrm{rad}}$, respectively) by the statistical significant differences between Plate $+4(+0.300)$ and Plate $+3(+0.150)$ and between all the other plates (except for Plate +3 versus Plate +1 on both conditions), both without and with tropicamide. Observing Table 1 and still having the baseline measure as reference, there are less significant differences under cycloplegia effect for the LDI, showing statistically significant differences between baseline visit and Plates +2 , Plate +3 , and Plate +4 (that induce higher positive SA). When the eye can accommodate, there are statistical significant differences between baseline and Plate -4 , Plate +1 , Plate +2 , Plate +3 , and Plate +4 .

\section{Discussion}

In this work, pupillary dilation and lack of accommodation caused by the effect of cyclopegia led to an increment in the size of the LD, when increasing values of positive SA were artificially induced to healthy eyes. This effect was not observed in the same amount when the eye could accommodate, which suggests that the accommodative mechanism and pupillary constriction are capable of compensating the degradation induced to the optical quality of the healthy eye.

The role of the pupil in the optical quality is well known as the higher order aberrations (HOA) increase and as the pupil dilates. This is more evident when HOA are induced with optical treatments such as orthokeratology or refractive corneal surgery. ${ }^{10}$ In fact, a larger pupil size under photopic conditions is a contraindication for those treatments. Despite this, the role of the pupil size as a main contributor to light disturbances is not so evident. Villa-Collar et al. ${ }^{11}$ have found a moderate but not significant positive correlation between the pupil size and the magnitude of the light disturbance in eyes undergoing myopic laser-assisted in situ keratomileusis (LASIK) surgery. The authors suggested that larger pupils were associated with stronger disturbances, but pupil size by itself was found to be only moderately correlated with this worsening effect in those eyes presenting positive SA as a consequence of the surgical procedure. More recently, Ferreira-Neves et al. ${ }^{9}$ evaluating nonoperated eyes showed that changing the pupil size from 3 to $6 \mathrm{~mm}$ did not have a significant impact on LD in healthy subjects with pharmacologically paralyzed accommodation. At first, this seems to imply that pupil size has no significant effect on light disturbance and accommodation might be involved. But this might not be entirely true. Accommodation might be responsible for maintaining the distortion index relatively low until the artificially induced SA was higher (0.300) as shown in Fig. 2(a). However, under tropicamide, the dilated pupil size makes the eye more sensitive to the induction of positive $\mathrm{SA}$, but not to the induction of negative SA, even for the higher values $(-0.300)$. This is interpreted by us as the pupil size having a greater impact on image quality for higher values of induced positive SA, under conditions of larger pupil dilatation such as night driving or night viewing of bright sources of light against a dark background. If the pupil size does not make any attenuation effect, the artificial induction of higher values of negative SA should degrade further the image quality of the source of light. Another explanation could be that the negative SA artificially presented to the eye under cycloplegia partially compensates the high positive SA found in these eyes under cycloplegia for the maximum round natural pupil. 


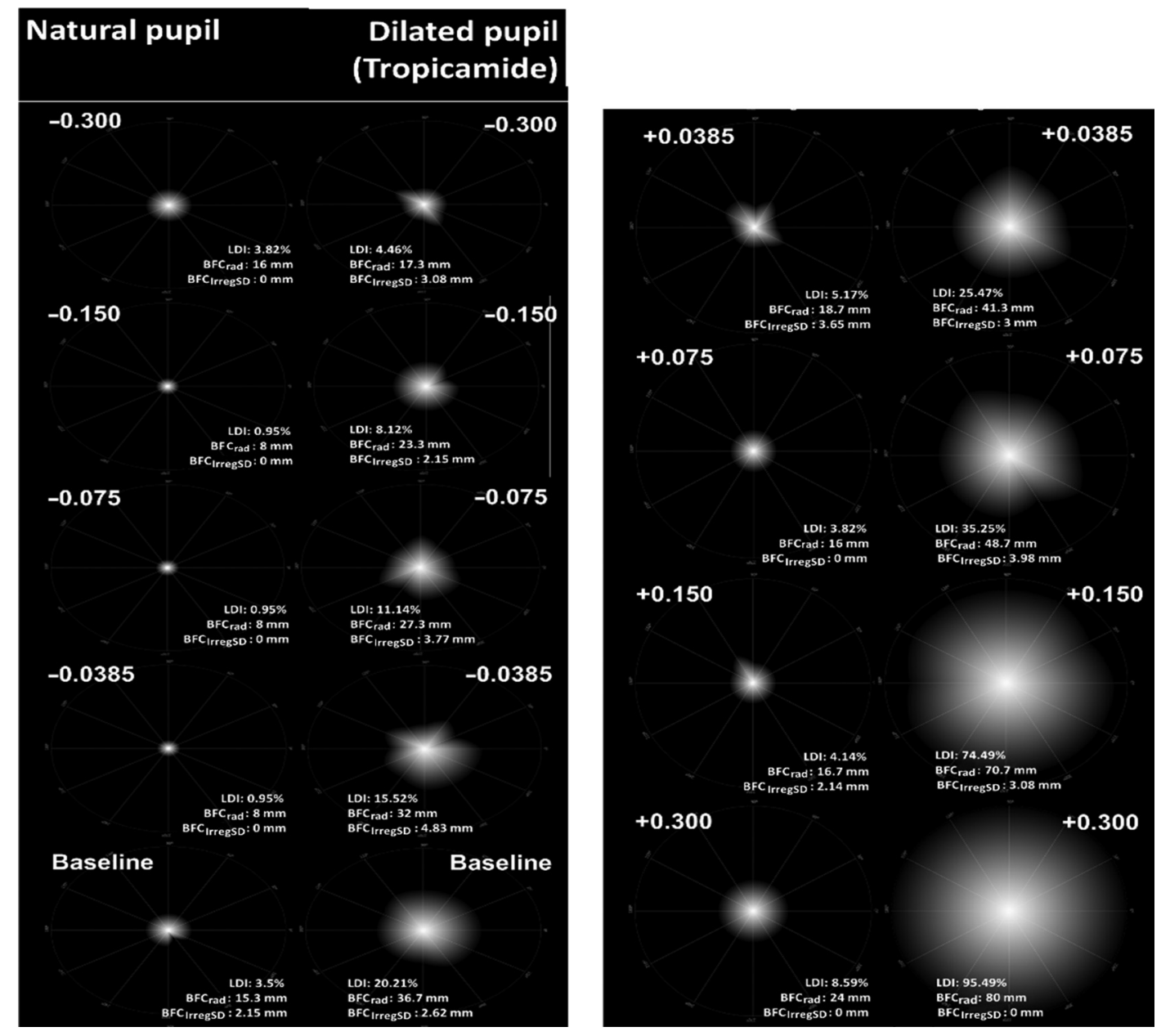

Fig. 3 Examples of graphical information of the size and shape of the LD observed through different plates without tropicamide and with tropicamide.

A recent research has evaluated the amount of subjective night myopia experienced by observers viewing a bright LED (0.5-mm diameter) against a dark source. Subjects were asked to adjust an optometer to reduce the disturbances surrounding the bright source of light, constituting a similar situation to that experience in this study with the LDA device. The investigators found that under such conditions, the eye tends to overaccommodate in order to minimize the disturbances surrounding the bright spot. The authors hypothesize that such "overaccommodative response" contributes to naturally reduce positive SA of the eye and/or shift the best focus to find the "smaller" point spread function. ${ }^{12}$ In fact, it is accepted that the SA reduces as the eye accommodates. ${ }^{13}$ López-Gil et al. hypothesized that a shift in the preferred focus of the eye driven by the search for the highest compactness of the point source of the stimulus would be the responsible for this change. However, in that experiment, the optometer was able to compensate for the defocus induced by the excessive accommodation response. ${ }^{12}$ In this study, an optometer was not available to compensate the defocus induced and we did not control the actual accommodative response except under cycloplegia. Thus, we can assume that pupil constriction and accommodation both contributed to reduce the distorting the image of the source of light induced by SA. This is in agreement with a more recent study conducted by Peixoto-de-Matos et al. (unpublished data) showing that when controlling the pupil size with an artificial diaphragm, eyes with active accommodation presented larger changes in the best focus than eyes with the accommodation paralyzed. This suggests that when accommodation is available, the eye changes its best subjective focus toward a more myopic situation when viewing a bright source of light against a dark background and this improves the contrast and compactness of the image, which is measured in this study. In result of the same experiment, the authors observed that this effect was of higher magnitude as the positive SA was artificially increased and was not observed when negative SA was induced, with or without the accommodation paralyzed and maintaining the pupil size constant through an artificial diaphragm. Their results suggest that inducing positive SA increases the light disturbances surrounding a source of light against a dark background, but this effect can be more efficiently attenuated when the accommodation is active than when accommodation is paralyzed, even for the same pupil size. The neural components cannot be overlooked in this sense as they might contribute to attenuation of the distortion effects induced by SA rendering lower values of distortion than might be expected. This neural adaptation is supposed to be present in patients undergoing refractive ${ }^{11}$ or cataract surgery. ${ }^{3}$ Also, Santolaria et al. found that LD is increased in ortho-k patients, in whom there is an increased positive SA induced by the treatment, and this effect decreases over the following months while aberrations maintain the same level. ${ }^{14}$ However, we consider that neural adaptation has no major effect 

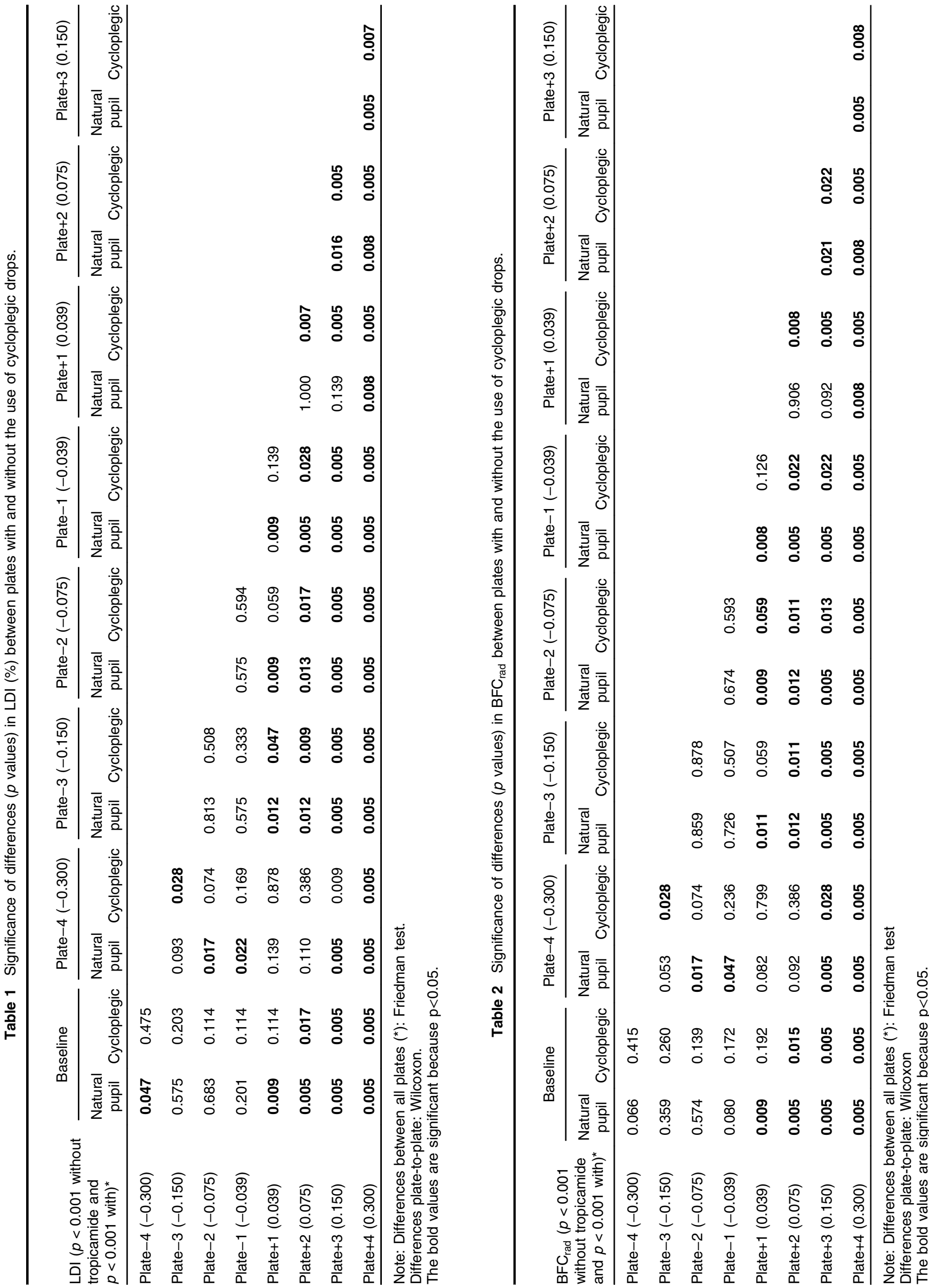


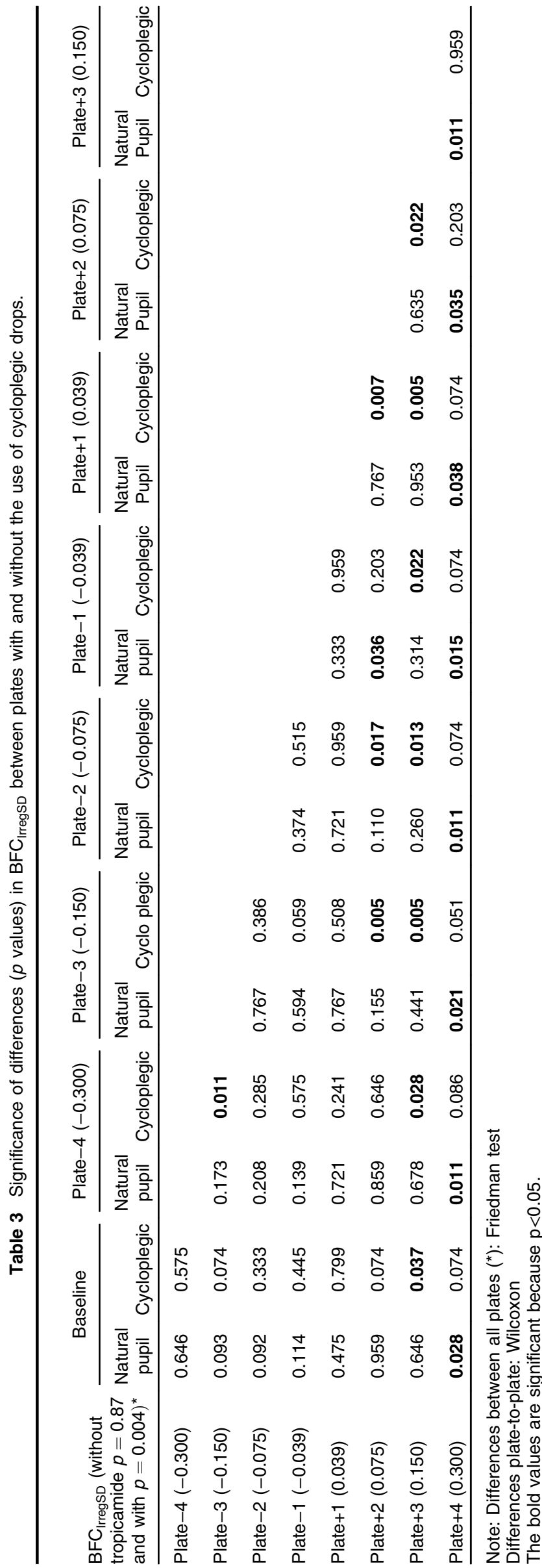

on this study due to the short term of presentation and duration of the experiment.

Finally, when the accommodation is paralyzed, the focus of the eye changes toward a more hyperopic situation, which might also increase the size of the LD. Altogether, this reinforces our hypothesis that absence of accommodation, rather than pupil dilatation alone is the responsible for the increased disturbance with increasing levels of SA in this study. However, this has to be evaluated in future studies by controlling the accommodation of the eye using an objective method and controlling the pupil size.

In this study, the induction of negative SA had no significant effects on the size of the LD or even caused an improvement in some subjects. This might be due to the inherent positive SA presented by the subjects with and without the cycloplegic effect. As mentioned before, for the maximum round pupil, we found a fourth-order SA of $+0.16 \pm 0.1 \mu \mathrm{m}$ without instillation of tropicamide and $+0.29 \pm 0.35 \mu \mathrm{m}$ before the instillation. This could be the reason why the phase plates that induce $-0.0385,-0.075$, and $-0.150 \mu \mathrm{m}$ seem to cause an improvement in LD size and shape.

All mentioned situations are shown in Fig. 3, by means of graphical information of the LD, and is a good and representative way to show what and how our subjects actually saw the source of light through different plates without and with cycloplegia.

There are some limitations in this study, such as the difficulty of centering the phase plates in the trial frame. Decentering could induce some coma aberrations and change the LDA results. However, during the measurements, this effect can be controlled by checking the position during the experiment and also verifying the location of the disturbance, i.e., a decentering effect would decenter the outcome light disturbance results toward an eccentric location, which was not observed in the images shown in Fig. 3. Another limitation is the fact that we do not exactly know the amount of SA induced by each plate. The values reported in Sec. 2 are valid for a 5-mm aperture. Larger pupils will be of course under higher levels of SA induced by these plates and affect differently the light disturbance phenomena measured. However, as the average pupil size was very similar among the different subjects, this effect would be similar for all the subjects evaluated. This could be expected as all the subjects had similar age and were evaluated under the same light conditions. Using an artificial pupil to limit the aperture would be of benefit to standardize the experiment. However, considering that all the plates were trialed in the same eyes, subjected to pharmacological pupil dilatation, the pupil aperture might be the same for all plates. Thus, we consider that the fact of not including an artificial pupil might be a limitation but avoids other undesirable effects motivated by decentration of the aperture against the pupil and should not impact the consistency of the comparisons between the different plates, which is the main goal of this study.

Finally, the reduced sample size is also a limitation, but the sample was powered enough to obtain statistically significant differences even after correction for multiple comparisons was applied. We emphasize that this study does not pretend to be a population-based study to test the effect of inherent SA of the eye on LD, but to evaluate the impact of artificially inducing SA of different degrees and signs on eyes with their particular SA values under cycloplegic and noncycloplegic conditions. Future studies with a larger sample and a major variety of refractive 
errors are needed to obtain data that can be representative of the general population. A larger scale study evaluating eyes with different amounts of aberrations might help to understand the effect of these optical imperfections in the LD. However, we need to consider that eyes having natural or surgically induced SA might present low values of distortion because of neural adaptation and it would be difficult to evaluate the effect of the optical components of aberrations on the LDs. Therefore, we still believe that in spite of the relevance of the larger clinical studies, studies such as the present one under well-controlled conditions and without neural adaptation taking part, is of benefit in understanding the short-term effects of aberrations on the eye, with particular relevance to better understanding the shortterm effects of surgical procedures.

In summary, this study showed that the LDA device is sensitive to changes in image quality caused by modifications in SA and might serve to simulate and quantify clinical and surgical conditions, where this aberration is altered (i.e., LASIK, orthokeratology, multifocal IOL, and so on).

\section{Acknowledgments}

This study was funded by the FEDER through the COMPETE Program and by the Portuguese Foundation for Science and Technology (FCT) in the framework of projects PTDC/SAUBEB/098391/2008, PTDC/SAU-BEB/098392/2008 and the Strategic Project PEST-C/FIS/UI607/2011. The authors thank the assistance of Professor Norberto López-Gil for the loan of the SA plates and the discussion of the results. R.M.A, J. M.G.M., H.F.N., L.R.V., and S.C.P.M have applied for a patent on the experimental device. Other authors declare that they do not have any proprietary or financial interest in any of the materials mentioned in this article.

\section{References}

1. N. I. Fan-Paul et al., "Night vision disturbances after corneal refractive surgery," Surv. Ophthalmol. 47(6), 533-546 (2002).

2. D. H. Lim et al., "Risk factors associated with night vision disturbances after phakic intraocular lens implantation," Am. J. Ophthalmol. 157(1), 135-141 (2014).

3. P. Brito et al., "Light-distortion analysis as a possible indicator of visual quality after refractive lens exchange with diffractive multifocal intraocular lenses," J. Cataract Refractive Surg. 41(3), 613-622 (2015).

4. S. D. Klyce, "Night vision disturbances after refractive surgery: haloes are not just for angels," Br. J. Ophthalmol. 91(8), 992-993 (2007).

5. D. Z. Reinstein, T. J. Archer, and M. Gobbe, "Is topography-guided ablation profile centered on the corneal vertex better than wavefrontguided ablation profile centered on the entrance pupil?" J. Refractive Surg. 28(2), 139-143 (2012).

6. J. J. Castro et al., "New testing software for quantifying discrimination capacity in subjects with ocular pathologies," J. Biomed. Opt. 16(1), 015001 (2011).

7. R. Gutierrez et al., "Simple device for quantifying the influence of halos after LASIK surgery," J. Biomed. Opt. 8(4), 663-637 (2003).
8. J. M. Linhares et al., "Radiometric characterization of a novel LED array system for visual assessment," J. Mod. Opt. 60, 1136-1144 (2013).

9. H. Ferreira-Neves et al., "Validation of a method to measure light distortion surrounding a source of glare," J Biomed. Opt. 20(7), 75002 (2015).

10. A. Queiros et al., "Effect of pupil size on corneal aberrations before and after standard laser in situ keratomileusis, custom laser in situ keratomileusis, and corneal refractive therapy," Am. J. Ophthalmol. 150(1), 97-109 (2010).

11. C. Villa-Collar et al., "Night vision disturbances after successful LASIK surgery," Br. J. Ophthalmol. 91(8), 1031-1037 (2007).

12. N. López-Gil et al., "Shedding light on night myopia," J. Vision 12(5), 4 (2012).

13. N. López-Gil et al., "Accommodation-related changes in monochromatic aberrations of the human eye as a function of age," Invest. Ophthalmol. Vis. Sci. 49(4), 1736-1743 (2008).

14. S. E. Santolaria et al., "Short-term changes in light distortion in orthokeratology subjects," Biomed. Res. Int. 2015, 278425 (2015).

Rute Macedo-de-Araújo concluded her bachelor in optometry and vision science in 2012 and completed the master degree in advanced optometry in 2014, both at University of Minho. Currently, she is a PhD student at CEORLab. Her research is focused on contact lenses, ocular surface, optical quality of the human eye, and light distortion. She has presented several communications in national and international congresses. She has coauthored two peer-reviewed papers and one book chapter.

Helena Ferreira-Neves concluded her bachelor in optometry and vision sciences in 2010 from the University of Minho and completed her master degree in advanced optometry. Currently, she is a PhD student in the CEORLab. She has participated in several research projects in the areas of light distortion, presbyopia, and optical solutions for its compensation. She is a coauthor of five papers and several communications in national and international scientific conferences.

Laura Rico-del-Viejo received a bachelor degree in optometry in 2012 at the University of Granada and is currently completing a master's degree in advance clinical optometry at the University of Minho. Currently, her research is focused on light distortion, multifocal contact lenses, orthoqueratology, biomechanical properties of the cornea, and contact lenses. She is a coauthor of 3 articles published and international scientific conferences.

Sofia C. Peixoto-de-Matos concluded her bachelor in optometry and vision science in 2008 and completed the master degree in advanced optometry in 2011 at University of Minho. She has presented in several national and international meetings and her current research is focused on contact lenses, myopia progression, and night myopia. She has coauthored several peer-reviewed papers and book chapters. At present, she coordinates a clinical trial related to the use of contact lenses to regulate myopia progression.

José Manuel González-Méijome graduated with a bachelor's degree in optics and optometry with honors from the University of Santiago de Compostela in 1997. He received his $\mathrm{PhD}$ in science at the University of Minho in 2007. He is currently an associate professor at the University of Minho and editor-in-chief of the Journal of Optometry. $\mathrm{He}$ has authored 125 articles indexed in ISI web of science with over 1300 citations, 3 books, and 15 book chapters. 